\title{
Experiences from Subspace System Identification - Comments from Process Industry Users and Researchers
}

\author{
Bo Wahlberg ${ }^{1}$, Magnus Jansson ${ }^{2}$, Ted Matsko ${ }^{3}$, and Mats A. Molander ${ }^{4}$ \\ 1 Automatic Control, KTH, Stockholm, Sweden, bo@ee.kth.se \\ 2 Signal Processing, KTH, Stockholm, Sweden, magnus.jansson@ee.kth.se \\ 3 ABB USA, ted.matsko@us.abb.com \\ 4 ABB Corporate Research, Västerås, Sweden, mats.a.molander@se.abb.com
}

Summary. Subspace System Identification is by now an established methodology for experimental modelling. The basic theory is well understood and it is more or less a standard tool in industry. The two main research problems in subspace system identification that have been studied in the recent years are closed loop system identification and performance analysis.

The aim of this contribution is quite different. We have asked an industrial expert working in process control a set of questions on how subspace system identification is used in design of model predictive control systems for process industry. As maybe expected, it turns out that a main issue is experiment/input design. Here, the difference between theory and practice is rather large mainly due to implementation constraints, but also lack of knowledge transfer. Motivated by the response from the expert, we will discuss several important user choices problems, such as optimal input design, merging of data sets and merging of models.

\section{Introduction}

System identification concerns the construction and validation of models of dynamical systems from experimental data. All advanced control and optimization methods, as model predictive control, require a reliable model of the process. System identification is thus a key technology for industry to benefit from powerful control methods. Modelling and system identification techniques suitable for industrial use and tailored for control design applications are of utmost importance The field of system identification is well developed with excellent textbooks, e.g. (Ljung, 1999; Söderström and Stoica, 1989), and software packages.

Subspace system identification is a class of methods for estimating state space models based on low rank properties of certain observability/prediction sets. The area was pioneered by researchers as Larimore, de Moor \& van Over- 
schee, Verhaegen and others, and was early adopted by industry. The theoretical foundation was established by for example Picci, Bauer and Jansson, and more recently by Chiuso. The industry was an early adopter. One of the first comercially available tools, released 1990, was ADAPTx from Adaptics Inc. It was based on the CVA method developped by Larimore. Nowadays, several vendors of Process Automation systems offer modeling tools based on subspace system identification. Examples are aspenOne from Aspen Technologies and Profit SensorPro from Honeywell.

ABB released its MPC product Predict \& Control in 2000 (called 3dMPC at the time). It uses state-space models combined with Kalman-style state estimators for the predicions. The included system identification package is based on a two-step procedure where an initial model is obtained through subspace system identification, and a refined model can be obtained through the minimization of a Prediction Error criterion.

Let us now review some basic facts about subspace system identification. The matrices of a linear system in state space form

$$
\begin{aligned}
x(t+1) & =A x(t)+B u(t) \\
y(t) & =C x t(t)+D u(t)
\end{aligned}
$$

can efficiently be estimated from observations of the input signal $u(t)$ and the output signal $y(t)$ without any prespecified parametrization using subspace system identification methods. The basic idea is that if the state vectors $x(t)$, and the input $u(t)$ and the output $y(t)$ are known, least squares linear regression techniques can be used to directly estimate the state-space matrices $\mathrm{A}, \mathrm{B}, \mathrm{C}$ and $\mathrm{D}$. The key observation is that the state $x(t)$ actually can be reconstructed from input output data using certain multiple-step ahead prediction formulas combined with low rank matrix factorizations. Combining these two ideas leads to a family of subspace system identification methods such as CVA, N4SID, and MOESP variations.

A great advantage of subspace system identification is that there is no difference between multi-input-multi-output system identification, where the input and output are vectors, and ordinary single-input single output identification. This is in contrast to e.g. prediction error methods, where the choice of model structures and corresponding parameterizations is most important. Other advantages of subspace identification are that the methods are based on robust numerical methods and avoid problem with optimization and possible local minima. The main disadvantages have been a potential performance loss compared to statistically efficient methods, as the prediction error method, and potential bias problems in identification of systems operated in closed loop. These two shortcomings have at least partly been eliminated by recent research (Jansson, 2003; Jansson, 2005; Chiuso and Picci, 2005; Qin et al., 2005).

There are mainly three design parameters in a subspace system identification method. We will here use the notation in (Ljung, 1999). The maximum 
prediction horizon is denoted by $r$, the number of past outputs used in the predictors is denoted by $s_{y}$, and the number of past inputs used in the predictors by $s_{u}$. A common choice is $s_{u}=s_{y}=s$. The model order $n$ is also important, but it is often estimated. It is often difficult to pre-specify $r$ and $s$. One common way in practice is to evaluate a set of combinations and then use model validation to determine the best values.

Subspace system identification was early used in industry since it fits very well with model predictive control. It is by now almost a standard technology and hence it is of interest to see how it is used in industry and how this relates to the state-of-the-art in more theoretical academic research.

\section{Questions and Answers from The User}

It is important to understand some of the more typical modelling problems in process industry. The plants are often very complex and it could be very expensive or even dangerous to do experiments with too large excitations. The process dynamics is slow, and the time constants are often around minutes or hours. This means that experiments often take long time. The process noise has often low frequency characteristics, e.g. drifts and random walks.

\section{Notation}

The notation $M V$ is used for Manipulated Variable, i.e. input signals which then will be used to control the systems. Control variables are denoted by $C V$, i.e. output signals which will be used for feedback control, while $P V$ denotes process variables i.e. measurable output signals.

We have formulated a set of questions to Ted Matsko, who is an expert at $\mathrm{ABB}$ in implementation of control systems, and, in particular, design of model predictive controllers using subspace system identification as a tool for modelling.

First, we think it would be interesting if you could put down some kind of step by step list of actions describing your typical routine for the modelling part of a model predictive control commissioning. Preferably also with time estimates (relative or absolute) for the different steps. Also, notes on steps where special care has to be taken to not run into problems would be very interesting.

Data Collection: This averages one day per MV for refining and petrochemical processes, which have longer time constants. Powerhouse is typically two MVs per day. There tends to be a greater level of unmeasured disturbance in refining/petrochem than in power, except for coal fired units where coal quality can vary. A typical experiment takes around 4 hours. 
The rest of the time concerns design of experiments and is often done in collaboration with an operator. A typical input signal is series of steps (4-8), which is manually implemented by the operator. Some of these are shorter than others and the amplitude and the sign may change.

Data review and organization: This typically takes 2-4 hours per data set. Typically we try to step one MV at a time. This never happens (operator needs to move another MV), so we need to organize the data and merge data files together. I have had bad experience with full MIMO ID using old tools from about 12 years ago. With a dense model matrix, the package simply did not work that well with a PRBS input. This may have worked better if the system had been more diagonal. I typically import data into an ABB tool and do a lot of plotting. Then I clean and merge data files in Excel, add calculated data points.

Subspace System Identification, first pass Specify a fairly small range of possible models by setting the design parameters to $r=s$ rather small and check different model orders. This usually gives around 150 models. Pick 3 or 4 with low open loop error, usually with different $n$. My experience is that higher model orders tend to result in a step response with oscillation. Evaluate against data set, look at step response (and impulse response if integrating process). Use prediction error plots to confirm what your eyes see.

Subspace System Identification, 2nd, 3rd ...passes If best models are on edge of $n / r / s$ boundaries, expand the range. Push to low $n$, very low $s$ and high $r$, gives best results in my experience.

Merge Models Paste things together with the Merge and Connect tools (ABB tool). Use model order reduction to reduce the order of the overall system. Sometimes we convert to Laplace transfer functions before merging. This gives us a little more control over the shape, dead time, gain, ability to zero out certain MV/CV pairs. May also run subspace system identification on $1 \mathrm{CV} \times 3$ or $4 \mathrm{MVs}$ to control, then merge to control placing of null relationships.

I like to break things down to /actuator-to-process output/ and /control loop-to-actuator/ models and then merge. Going actuator-to-output provides a physical, intuitive model. Sometimes the base controls can cloud the issue and hide a bad job from visual inspection.

Modeling and Constraint design are the two most important steps in creating good MPC applications. Our subspace system identification tool is the best modeling tool I have used. Our Data Processing tools are almost good, but the latest release makes them difficult to use in some circumstances. Data ma- 
nipulation and pre-processing can consume a lot of time if you make mistakes in data collection.

How do you set up the identification experiments? Do you try to disturb as many as possible of the MVs simultaneously? If not all MVs are disturbed simultaneously, what considerations control the splitting of the experiments? How do you typically decide the step amplitudes for the excitation?

We first create a large taglist. It is better to collect any data related to the process. If you find some inexplicable behavior, going through data for ancillary tags may allow you to save the data file and avoid doing additional tests. Additional tests may require another trip to site. We try to step one MV at a time. If the process is large and we can step variables that do not overlap in the model matrix, we may step two at the same time. An important part of working with any modeling package is looking at how well the model fits the data visually. In a full 5x5 MIMO ID with all MVs moving randomly, it is very hard to tell how good a model fit really is. To determine step test amplitudes, we monitor the noise level and talk to the operators about the process gains and settling time. The operator will be able to give you a reasonable feel for the gain and time constant. As a minimum, I want the step tests to move the PV twice as far as the noise does. After a few steps, we may adjust.

Are the identification experiments always performed in open-loop, or is it common to have the MV excitations as additions to the outputs of a running controller? If you commonly perform both types of experiments, what are your experience of the differences?

Before commissioning, most data is open loop. Infrequently the MPC will include a distillation column temperature that is controlled in the distributed control system (DCS) with a cascade loop. In the powerhouse, the pressure is always controlled. In these cases we may do some or all of the initial testing with that $\mathrm{PV}$ in closed loop. To fine tune a model during commissioning, we may use some closed loop data. I do not have a lot of experience with closed loop data and subspace system identification, but the header pressure models seem to work better when at least some of the data is under closed loop control. That may be a special case where the models are integrating (or very long time constants). I seem to recall that other model predictive control system identification packages discouraged the use of closed loop data.

Do you find the time needed for the identification experiments to be a main hurdle?

Data collection time is about $1 / 3$ of the total time spent on building dynamic models. Sometimes we are able to get people at the plant to do some of the data collection under our guidance. There is also time for functional spec- 
ification, configuring controller, configuring DCS, adding DCS graphics, FAT, commissioning, final documentation. So data collection time is significant, but not the majority of time.

Compared to other MIMO control systems you commissioned earlier, do you save a lot of time in the modelling phase by using the subspace system identification tools? If so, in what stages of the modelling, and how much?

Time is about the same. Quality is better and that saves time commissioning. Capability and flexibility is better (merge, connect). Better models make commissioning much easier. Biggest place for time savings would be improving our data preprocessing tools. We handle some big data sets, 100 tags by 17,500 records is a typical file (merged 4 days testing into one file in this example).

In the ABB modelling tool we have the two step procedure of a Subspace identification, followed by a Prediction Error Model identification. How important is the second PEM step? Is it always important, or have you identified situations where it is not important and others where it is? (Theoretically, one would suspect that it is more important if the identification experiment was performed in closed loop)?

I have not found that the PEM step makes much improvement. The feature to force a zero relationship does work that well (I have a recent example).

How about size restrictions? How large problems do you typically model, in number of model inputs/outputs? When do you normally run into problems regarding size?

I have had no problem with MV x PV size, but I do not stretch the limits. Even with a big controller, the biggest subset I work with is around $4 \mathrm{x} 4$. I use some very long files with breaks in time. This may make the subspace system identification slow, but the results are usually good. When I plot the model predictions when using data sets with breaks, I still get some strange initialization occasionally, but I am not very well educated on how the calculations work here so user error is not unlikely.

How large models (in number of states) do you normally end up with? Is it very coupled to the input/putput size, or do you normally find models with a reasonably small number of states even if the number of inputs/outputs are large?

I usually end up with models that have a low number of states from subspace system identification. Some of the models used for control, that are merged, are pretty large. On the small powerhouse project we just finished, 
individual models were $n=2$ to 6 . final merge was $n=44$ ( $5 \mathrm{PVs} \times 4 \mathrm{MVs}$ ). On a prior project with many MVs, I had a plant model with $n=166$, but the order reduction tool pared that to 65 .

How do you normally conclude that a model is good enough? What evaluation methods are most important?

Visual examination of prediction versus actual data. Of course if you made some mistakes that can still look good, even if the model is not. If you have problems commissioning, the first thing to re-examine are the models. There we take new closed loop data files and import them into the identification tool and look at the predictions versus actual output.

Do you consider the existing evaluation tools sufficient, or do you sometimes experience that a model that looks perfectly good, still does not work well for the $M P C$ ?

Yes, as I said above this happens. Usually it is some user error. Something that one overlooks in the data file. Recent example was a delay on one signal due to the communication system in the DCS. The delay did not exist for process control, just data collection. All my models were slightly off, but gains were good. This caused a nice limit cycle.

Do you always use separate identification and evaluation data sets, or do you sometimes/occassionally/always use the same data set for both identification and evaluation?

Always use the same in the identification step. Sometimes I will use another file for plotting predictions, usually data gathered from a later time period. Unless we start to do long PRBS runs, I don't think there is typically enough data to split.

As a last point, do you have specific wishes for improvements of the modelling tools?

Better results from the PEM step (a.) gains set to 0 ( b.) conversion, Order reduction tool introduces some small gains where 0 gains may have existed before. A tool to convert to low order Laplace transfer functions from state space models. An online tool to monitor model performance.

\section{Comments from the Researchers}

The responses and answers from the process control expert are very interesting and open up a lot of very interesting research issues. Our objective, however, 
is not to provide a "solutions manual," but instead to relate the comments of the expert to the current status in research. We are well aware of the difficult trade-offs in real applications, and that academic examples often are severe simplifications. However, they can provide insight and results which then can be tailored real problems to the intended application. We will, in particular, discuss input design, merging of data sets and merging of estimated models.

\section{Input Design}

Input design for linear dynamic systems started out around 1960 and in the 1970's there were a lot of activities in this area, for which (Goodwin and Payne, 1977) acts as an excellent survey. The classical approach is to minimize some scalar measure function of the asymptotic parameter covariance matrix (e.g. the determinant or the trace) with constraints on input and/or output power.

Let $\Phi_{u}$ be the power spectral density of the input. The more recent approaches to optimal experiment design problems are typically posed in the form

$$
\min _{u} \frac{1}{2 \pi} \int_{-\pi}^{\pi} \Phi_{u}(\omega) d \omega
$$

subject to model quality constraints, and signal constraints

i.e., formulated as optimization problems that include some constraints on the model quality together with signal constraints. It is also possible to minimize a weighted version of the input signal or the output signals. The signal constraints have to be included to obtain well-posed problems, e.g., to prevent the use of infinite input power. Powerful methods have been developed to solve a variety of such problems. Notice that the solution will be an input spectral density, and that the time domain realization problem then can be solved in different ways. We refer to (Jansson, 2004; Barenthin, 2006; Barenthin et al., 2006) for overviews of recent methods.

System identification for control is a very important application of input design. Here the model quality constraints reflect robust performance and stability. It is very important to obtain input signals that then can be reliably implemented, while the optimal solution often only refers to frequency domain properties. To stress this issue (Rivera et al., 2003) have introduced the concept of "plant-friendly" identification.

\section{Optimal Input Design for Subspace Methods}

Most of the input design methods make use of asymptotic variance analysis based on the Prediction Error identification Method (PEM) framework, (Ljung, 1999). Very few results have been presented for optimal input design 
for subspace system identification methods. A natural idea would be to do input design for PEM methods and then apply it for subspace system identification. Our conjecture is that optimal inputs for PEM would give good results for subspace system identifications methods since subspace methods often give results with quality properties close to those of PEM.

From theory it is known that subspace system identification requires that the input signal is persistently exciting of order $n+r$ or higher (Jansson, 1997; Chui and Maciejowski, 2005), and that white noise input signals often give good performance. This is in contrast to the experiments described by the expert, which essentially are of step response type. The signal to noise ratio is, however, quite high. It would be of interest to do performance analysis of subspace system identification model estimates from step response data with high signal to noise ratio. Of course, a noise free step response contains all information about a linear system, but it is very sensitive to disturbances. Early subspace system identification techniques made use of the Hankel matrix constructed from impulse response data.

An interesting input design problem is the following. Assume that you are only allowed to change the input signal $d$ times over a given interval with prespecified amplitude levels. What are the optimal choices of times to change the signal?

\section{Example}

Some insight can be obtained by the following simple example. Consider the problem of estimating the parameters of a first order ARX model

$$
y(t)=-a y(t-1)+b u(t-1)+e(t), \quad a=0.5, b=1
$$

under the assumption of zero initial conditions and only the choices $u=1$ or $u=-1$ are allowed and that one only is allowed to change the input from 1 to -1 once. Assume that you have four measured samples. Possible input sequences are then $(1,-1,-1,-1),(1,1,-1,-1),(1,1,1,-1)$ and $(1,1,1,1)$. Since this concerns very small data records, we have used Monte Carlo computer simulations to study the quality of the estimated parameters. For the estimation of $a$, it turns out the first sequence is the best one and it gives a performance that is almost twice as good as the second best one. The accuracies of the estimates of $b$ are approximately the same for the first and the third sequences. The fourth sequence is of course bad in terms of persistence of excitation. The example shows that it is important to design the input sequence even if one has hard constraints.

\section{MIMO Systems}

Identification of MIMO systems has recently been an active area of research. In particular variance analysis and input design issues. A main question is 
if one should excite the various inputs simultaneously or separately? From a pure performance perspective it is always better to excite all inputs simultaneously (Gevers et al., 2006). However, in the case where the output power is constrained, the conclusion is that it is possible to obtain nearly the same performance by exciting one input at a time, i.e. the strategy used by the expert. See page 25 in (Gevers et al., 2006).

In case the process has strong interactions, i.e. ill conditioned systems, the dynamics of the high-gain directions tends to dominate the data and it is difficult to estimate a reliable model for the low-gain direction. This problem is discussed in e.g. (Jacobsen, 1994). An interesting approach for iterative input design of gene regulatory networks is described in (Nordling and Jacobsen, 2006). The idea is to excite the inputs to obtain a uniform output excitation. It is a good idea to pre-compensate the plant to make it as diagonal as possible before identification. For a completely diagonal plant the MIMO identification problem of course simplifies to a number of SISO problems. It is of course difficult to do pre-compensation without a good model, and it could also create problems with large inputs if the plant is ill conditioned. A better solution is then to use partial decoupling as e.g. described in (Weller and Goodwin, 1996). The idea is to transform the system to a triangular structure.

\section{Merging data}

Assume that data have been collected from a series of SIMO experiments. The problem of merging data sets is discussed in details in Section 14.3 in (Ljung, 1999). One important aspect is that it is common that one has to remove parts of bad data, which means that data records have to then be merged.

For linear dynamical systems the main problem when merging data is to take the different initial conditions into account. In case of periodic input signals it is possible to use averaging as a means to merge data.

We will illustrate some of the issues by a simple example.

\section{Example}

Consider the model

$$
y(t)=b u(t-k)+e(t)
$$

i.e. a time delayed linear relation with unknown gain $b$. Assume that we have two data sets of equal length

$$
\left\{y_{1}(t), u_{1}(t), t=1,2, \ldots, N / 2\right\}, \quad\left\{y_{2}(t), u_{2}(t), t=1,2 \ldots, N / 2\right\}
$$

The least squares (PEM) estimate minimizes 


$$
\min _{b} \sum_{t=1+k}^{N / 2}\left(y_{1}(t)-b u_{1}(t-k)\right)^{2}+\sum_{t=1+k}^{N / 2}\left(y_{2}(t)-b u_{2}(t-k)\right)^{2}
$$

and thus equals

$$
\hat{b}_{N}=\frac{\sum_{t=1+k}^{N / 2}\left(y_{1}(t) u_{1}(t-k)+y_{2}(t) u_{2}(t-k)\right)}{\sum_{t=1+k}^{N / 2}\left(u_{1}^{2}(t-k)+u_{2}^{2}(t-k)\right)}
$$

Notice that this does not correspond to just concatenating the data

$$
\begin{aligned}
& y_{c}(t)= \begin{cases}y_{1}(t) & t=1, \ldots, N / 2 \\
y_{2}(t-N / 2), & t=N / 2+1, \ldots, N\end{cases} \\
& u_{c}\left(t= \begin{cases}u_{1}(t), & t=1, \ldots, N / 2 \\
u_{2},(t-N / 2), & t=N / 2+1, \ldots, N\end{cases} \right.
\end{aligned}
$$

which would yield

$$
\hat{b}_{N}^{c}=\frac{\sum_{t=1+k}^{N} y_{c}(t) u_{c}(t-k)}{\sum_{t=1+k}^{N} u_{c}^{2}(t-k)} \neq \hat{b}_{N}
$$

since the transient has to be taken into account.

An observation is however that in case $u_{1}=u_{2}$, i.e. the same input is used in both experiments, we have

$$
\hat{b}_{N}=\frac{\sum_{t=1+k}^{N / 2}\left(y_{1}(t)+y_{2}(t)\right)\left(u_{1}(t-k)+u_{2}(t-k)\right)}{\sum_{t=1+k}^{N / 2} 0.5\left(u_{1}^{2}(t-k)+u_{1}^{2}(t-k)\right)}
$$

which just corresponds to adding the signals $y=y_{1}+y_{2}$ and $u=u_{1}+u_{2}$ and then use the ordinary PEM estimate

$$
\hat{b}_{N}=\frac{\left.\sum_{t=1+k}^{N / 2} y(t) u(t-k)\right)}{\sum_{t=1+k}^{N / 2} u^{2}(t-k)}
$$

The summation approach only works for repeated input signals and will help reducing noise contributions by averaging. Notice that we only work with data records of length $N / 2$ instead of $N$ when concatenating the data. This result holds for general models with repeated signals.

\section{Merging of Models}

It is easy to combine parameter estimates from different data sets by assuming that they can be regarded as independent. The minimum variance estimate is obtained by weighting the individual estimates with their inverse covariance 
matrix, see Equation (14.15) in (Ljung, 1999). It is also noted that the same result can be obtained by solving a combined least squares problem using the merged set of data.

Expression (4) in the example in the previous section illustrates exactly this. The least squares estimate from the individual data records equals

$$
\hat{b}_{N / 2}^{1}=\frac{\sum_{t=1+k}^{N / 2}\left(y_{1}(t) u_{1}(t-k)\right)}{\sum_{t=1+k}^{N / 2} u_{1}^{2}(t-k)}, \quad \hat{b}_{N / 2}^{2}=\frac{\sum_{t=1+k}^{N / 2}\left(y_{2}(t) u_{2}(t-k)\right)}{\sum_{t=1+k}^{N / 2} u_{2}^{2}(t-k)}
$$

with covariances

$$
\operatorname{Cov} \hat{b}_{N / 2}^{1}=\frac{\lambda_{o}}{\sum_{t=1+k}^{N / 2} u_{1}^{2}(t-k)}, \quad \operatorname{Cov} \hat{b}_{N / 2}^{2}=\frac{\lambda_{o}}{\sum_{t=1+k}^{N / 2} u_{2}^{2}(t-k)}
$$

Hence, the combined minimum variance estimate becomes

$$
\hat{b}_{N}=\frac{\hat{b}_{N / 2}^{1} / \operatorname{Cov} \hat{b}_{N / 2}^{1}+\hat{b}_{N / 2}^{2} / \operatorname{Cov} \hat{b}_{N / 2}^{2}}{1 / \operatorname{Cov} \hat{b}_{N / 2}^{1}+1 / \operatorname{Cov} \hat{b}_{N / 2}^{2}}
$$

which equals (4). The observation is that it is most important to take the quality of the estimates into account when merging different models. Hence, it is not trivial to combine model estimates from subspace system identification methods, and the best idea seems to be to instead merge the data sets. One problem is that the state space realization of the estimate depends on the data, and hence it is not just to combine the different state space matrices. Instead one has to work with canonical forms such as transfer functions or frequency responses.

Consider the problem of merging two frequency response estimates $\hat{G}_{1}\left(e^{i \omega}\right)$ and $\hat{G}_{2}\left(e^{i \omega}\right)$, possibly of different orders, estimated by e.g. subspace methods. Assume that the corresponding variances of the two estimates are given by $W_{1}\left(e^{i \omega}\right)$ and $W_{2}\left(e^{i \omega}\right)$, respectively. These variance expressions can be quite complicated to obtain. However, a reasonable approximation based on the high model order theory of Ljung (Ljung, 1999), is

$$
W_{i}=\frac{n_{i}}{N_{i}} \frac{\widehat{\Phi}_{v}^{i}\left(e^{i \omega}\right)}{\widehat{\Phi}_{u}^{i}\left(e^{i \omega}\right)}, \quad i=1,2
$$

where $\widehat{\Phi}_{v}^{i}$ is an estimate of the noise power spectrum, $\widehat{\Phi}_{u}^{i}$ is an estimate of the input power spectrum, $n_{i}$ is the model order and $N_{i}$ is the number of data used in experiment $i$.

The optimally merged estimate will then be

$$
\hat{G}_{3}=\frac{\hat{G}_{1} / W_{1}+\hat{G}_{2} / W_{2}}{1 / W_{1}+1 / W_{2}}
$$


with resulting variance $W_{3}=1 /\left(1 / W_{1}+1 / W_{2}\right)$. In order to obtain a low order transfer function estimate the following $\mathrm{H}_{2}$ model reduction problem can be solved

$$
\min _{\theta} \frac{1}{2 \pi} \int_{-\pi}^{\pi} \frac{\left|\hat{G}_{3}\left(e^{i \omega}\right)-G\left(e^{i \omega}, \theta\right)\right|^{2}}{W_{3}\left(e^{i \omega}\right)} d \omega
$$

where $G\left(\left(e^{i \omega}, \theta\right)\right.$ is a set of parameterized transfer functions. This approach was proposed in (Wahlberg, 1989) and has more recently been studied in (Tjärnström, 2002).

Further insight can be obtained if the noise properties in both experiments are identical and the model orders $n_{1}=n_{2}$. Let $\hat{\Phi}_{y u}^{i}=\hat{G}_{i} \widehat{\Phi}_{u}^{i}$, i.e. an estimate of the cross-spectra. Then

$$
\hat{G}_{3}=\frac{N_{1} \hat{\Phi}_{y u}^{1}+N_{2} \hat{\Phi}_{y u}^{2}}{N_{1} \hat{\Phi}_{u}^{1}+N_{2} \hat{\Phi}_{u}^{2}}
$$

which can be viewed as averaging the individual spectra

$$
\hat{\Phi}_{y u}^{3}=\frac{N_{1} \hat{\Phi}_{y u}^{1}+N_{2} \hat{\Phi}_{y u}^{2}}{N_{1}+N_{2}}, \quad \hat{\Phi}_{u}^{3}=\frac{N_{1} \hat{\Phi}_{u}^{1}+N_{2} \hat{\Phi}_{u}^{2}}{N_{1}+N_{2}}
$$

and then taking $\hat{G}_{3}=\hat{\Phi}_{y u}^{3} / \hat{\Phi}_{u}^{3}$. From a non-parametric estimation point of view this makes a lot of sense. Even further insight can be obtained by the following simple example. Let

$$
y_{3}=y_{1}+y_{2}, \quad u_{3}=u_{1}+u_{2}
$$

and assume that $u_{1}$ and $u_{2}$ are independent. Then

$$
\Phi_{y_{3} u_{3}}=\Phi_{y_{1} u_{1}}+\Phi_{y_{2} u_{2}}, \quad \Phi_{u_{3}}=\Phi_{u_{1}}+\Phi_{u_{2}}
$$

for the cross spectra and the input spectra. Hence, the merging of models corresponds in some sense to merging of data, while enforcing the independence assumption.

\section{Combining Sub-Models}

Consider the following problem

$$
\begin{aligned}
& z_{1}(t)=G_{1}(q) u(t) \\
& z_{2}(t)=G_{2}(q) z_{1}(t) \\
& y_{1}(t)=z_{1}(t)+e_{1}(t) \\
& y_{2}(t)=z_{2}(t)+e_{2}(t)
\end{aligned}
$$

Given observations of the input $u(t)$ and the outputs $y_{1}$ and $y_{2}$ we would like to estimate the transfer functions $G_{1}$ and $G_{2}$. There are at least three different 
ways to approach this problem. The optimal way is to use this structure in a single input two outputs (SITO) prediction error identification method. Another way is to estimate a state space model with one input and two outputs using subspace identification. The transfer functions $G_{1}$ and $G_{2}$ can then be estimated from the state space model estimates. A problem is, however, that the order of these two transfer functions will in general be of the same order as the total system. One way is to apply a model order reduction method, but then it is important to take the statistical properties of the model estimates into account.

As mentioned by the expert a common approach is to estimate individual sub-models, i.e. $G_{1}$ from observations of $u$ and $y_{1}$ and $G_{2}$ as the transfer function from $y_{1}$ to $y_{2}$. The second problem is an error-in-variables problem, see (Söderström, 2007), and care has to be taken in order to avoid problems caused by the noise $e_{1}$. In case the signal $z_{1}$ is of low frequency character or have a special structure due to the choice of input $u$ this information can be used to pre-process the signal before applying the identification method.

A third way is to estimate the transfer function $G_{1}$ from $u$ and $y_{1}$ and then $G_{3}=G_{1} G_{2}$ from $u$ to $y_{2}$. The transfer function $G_{2}$ can then be obtained as $\hat{G}_{2}=\hat{G}_{3} / \hat{G}_{1}$, but will in general be of high order and could also be unstable due to non-minimum phase zeros. A better solution is to solve

$$
\min _{G_{2}} \frac{1}{2 \pi} \int_{-\pi}^{\pi} \frac{\left|\hat{G}_{3}\left(e^{i \omega}\right)-\hat{G}_{1}\left(e^{i \omega}\right) G_{2}\left(e^{i \omega}\right)\right|^{2}}{W_{3}\left(e^{i \omega}\right)} d \omega
$$

where the weighting $W_{3}\left(e^{i \omega}\right)$ should reflect the variance of the error. One way is to calculate

$$
\operatorname{Cov} \frac{\hat{G}_{3}\left(e^{i \omega}\right)}{\hat{G}_{1}\left(e^{i \omega}\right)}=W_{4}\left(e^{i \omega}\right)
$$

and use $W_{3}\left(e^{i \omega}\right)=\left|\hat{G}_{1}\left(e^{i \omega}\right)\right|^{2} W_{4}\left(e^{i \omega}\right)$. This is very closely related to indirect PEM discussed in (Söderström et al., 1991).

The conclusion is that one should take the statistical properties of the model estimates into account when merging and combining sub model estimates.

\section{Conclusion}

The objectives of this paper have been to study how subspace system identification can be used in industrial applications and the corresponding supporting theoretical results. One very interesting area is input signal design, and in particular methods that take practical experiences and limitations and into account. Based on the input from an industrial user, we have discussed some theoretical results that can be useful for merging of data and models. 


\section{References}

Barenthin, M., H. Jansson, H. Hjalmarsson, J. Mårtensson and B. Wahlberg (2006). A control perspective on optimal input design in system identification. In: Forever Ljung in System Identification. Chap. 10. Forever Ljung in System Identification, Studentlitteratur.

Barenthin, Märta (2006). On input design in system identification for control. Technical report. Royal Institute of Technology (KTH). Licentiate Thesis.

Chiuso, A. and G. Picci (2005). Prediction error vs. subspace methods in open and closed-loop identification. In: 16th IFAC World Congress. Prague, Czech Republic.

Chui, N. L. C. and J. M. Maciejowski (2005). Criteria for informative experiments with subspace identification. International Journal of Control 78(5), 326-44.

Gevers, M., L. Miskovic, D. Bonvin and A. Karimi (2006). Identification of multiinput systems: variance analysis and input design issues. Automatica 42(4), 559572 .

Goodwin, G. and R.L. Payne (1977). Dynamic System Identification: Experiment Design and Data Analysis. Academic Press. New York.

Jacobsen, E. W. (1994). Identification for control of strongly interactive plants. In: AIChE Annual Meeting.

Jansson, H. (2004). Experiment design with applications in identification for control. PhD thesis. KTH. TRITA-S3-REG-0404.

Jansson, M. (1997). On Subspace Methods in System Identification and Sensor Array Signal Processing. PhD thesis.

Jansson, M. (2003). Subspace identification and ARX modeling. In: IFAC Symp. on System Identification. Rotterdam, The Netherlands.

Jansson, M. (2005). A new subspace identification method for open and closed loop data. In: 16th IFAC World Congress. Prague, Czech Republic.

Ljung, L. (1999). System Identification - Theory For the User, 2nd ed. PTR Prentice Hall. Upper Saddle River, N.J.

Nordling, T. E. M. and E. W. Jacobsen (2006). Experiment design for optimal excitation of gene regulatory networks. Poster.

Qin, Joe S., Weilu Lin and Lennart Ljung (2005). A novel subspace identification approach with enforced causal models. Automatica 41(12), 2043-2053.

Rivera, D.E., H. Lee, M.W. Braun and H.D. Mittelmann (2003). Plant-friendly system identification: a challenge for the process industries. In: Proceedings of the 13th IFAC Symposium on System Identification. Rotterdam, The Netherlands. pp. $917-922$.

Söderström, T. (2007). Errors-in-variables methods in system identification.. Automatica. Survey paper, to appear.

Söderström, T. and P. Stoica (1989). System identification. Prentice Hall International. Hertfordshire, UK.

Söderström, T., P. Stoica and B. Friedlander (1991). An indirect prediction error method. Automatica 27, 183-188.

Tjärnström, F. (2002). Variance Expressions and Model Reduction in System Identification. $\mathrm{PhD}$ thesis.

Wahlberg, B. (1989). Model reduction of high-order estimated models: The asymptotic ML approach. International Journal of Control.

Weller, S.R. and G.C. Goodwin (1996). Controller design for partial decoupling of linear multivariable systems. Int. J. Control 63(43), 535-556. 